\title{
Perioperative anaemia management in elective colorectal surgery
}

\author{
S. Sanz Serrano, C. Galeano Solís, L. Alonso Aguilar, M. Barbero Mielgo, D. Carballo \\ López, J. García Fernández.
}

\section{INTRODUCTION}

Anaemia is one of the most frequent extraintestinal manifestations of colorectal cancer with a prevalence up to $25 \%$ of moderate or severe anaemia. This condition has been linked to increased postoperative mortality and morbidity and allogeneic blood transfusion (ABT) ${ }^{1}$. Inappropriate transfusion practices may endanger patients and waste resources. Iron deficiency is the principal cause of anaemia. This situation makes it necessary to look out for new strategies in the management of perioperative anaemia. We evaluate the role of postoperative iron therapy (PIT) in the immediate postoperative period as part of a new multidisciplinary project of Enhanced Recovery After Surgery (ERAS).

\section{MATERIALS AND METHODS}

We review two cohorts: Prospective ERAS group and a retrospective NO ERAS group.

ERAS: 177 patients (108 men/ 69 women), mean age was 67.54 \pm 12.34 between January and November 2017 under ERAS program.

No ERAS: 66 (41 men/ 25 women), mean age was $65.8 \pm 13.88$ patients between July and October 2015, prior protocol establishment.

Patients under the ERAS program received $300 \mathrm{mg}$ of iron sucrose intravenously the day of surgery and the second day. We evaluate hemoglobin $(\mathrm{Hb})$ on the first, third and fifth days after surgery and the percentage of patients requiring transfusion in the postoperative period.

\section{RESULTS}

ERAS : laparoscopic approach 65\%, PIT 82\%

NO ERAS : laparoscopic approach 59\%, PIT 0\%

laparoscopic $p=0.136$, PIT $<0.001$

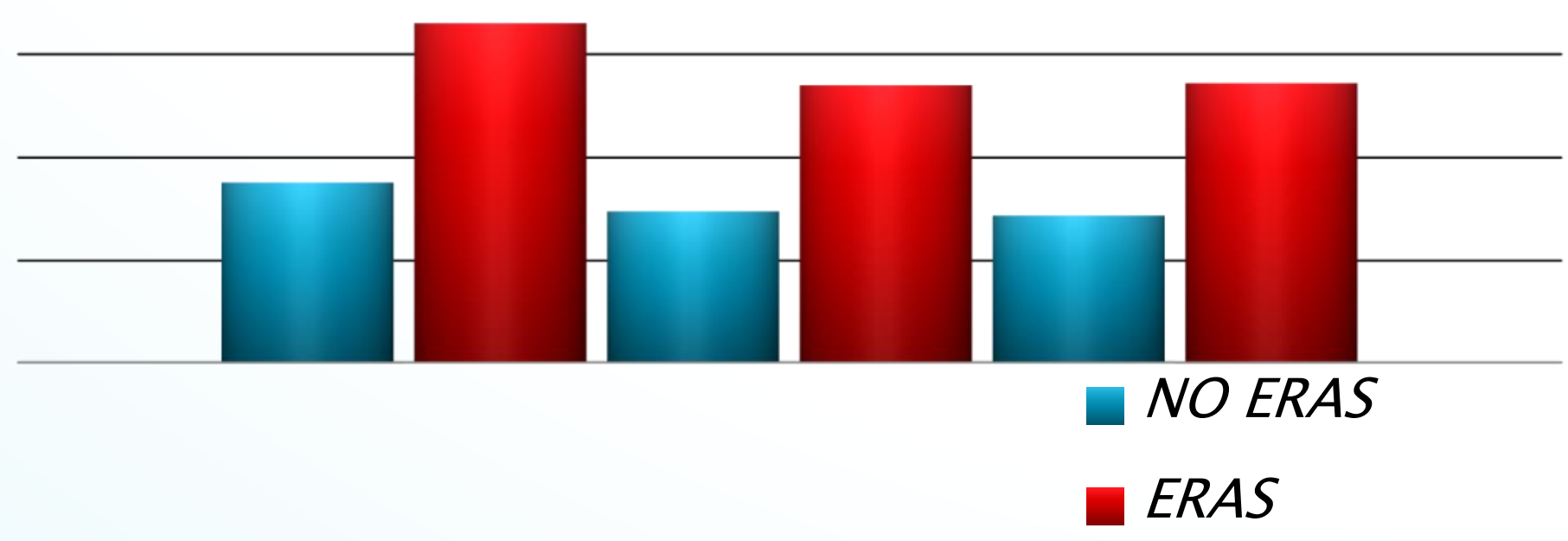

\section{CONCLUSION}

In the ERAS group we found higher hemoglobin levels significantly compared to the control group, in addition we can also find a large decrease in the percentage of patients who required to be transfusedy. PIT could be an effective treatment to decrease incidence of postoperative anaemia after colorectal surgery. We must wait up to three weeks to observe the maximum effect of iron sucrose, so it would be interesting to observe this trend in a similar period of time for future studies. Incidence of ABT was less frequent in ERAS group. This strategy of anaemia management could avoid risk factors associated to $A B T$.

\begin{tabular}{|c|c|c|c|}
\hline & NO ERAS & ERAS & $p$ \\
\hline$H b \mathrm{~g} / \mathrm{dl} 1^{\text {st }}$ day & $10,88 \pm 1,86$ & $11,65 \pm 2,25$ & 0,012 \\
\hline $\mathrm{Hb} g / d l 3^{r d} d a y$ & $10,74 \pm 1,76$ & $11,35 \pm 2,23$ & 0,091 \\
\hline$H b \mathrm{~g} / \mathrm{dl} 5^{\text {th }}$ day & $10,82 \pm 1,73$ & $11,36 \pm 1,95$ & 0,045 \\
\hline$\%$ postoperative $A B T$ & $21 \%$ & $5 \%$ & $<0.001$ \\
\hline
\end{tabular}

BIBLIOGRAPHY : Muñoz M, Gomez-Ramirez S, Martin- Montañez E y Auerbach M Perioperative anemia management in colorectal cancer patients: a pragmatic approach World J. Gastroenterology Feb 28;20(8):1972-85 2 Muñoz M, Naveira E, Seara J, Palmer J H, Cuenca J y García-Erce J.A. Role of parenteral iron in transfusion requirements after total hip replacement. A pilotstudy. Transfusion Medicine, 2006, 16, 137-142 